\title{
La participación efectiva de docentes y directivos docentes de instituciones educativas en la gestión integral del sistema educativo de Bogotá*
}

\section{The effective participation of teachers and senior teachers of educational institutions in the integral education management system in Bogotá}

Recibido: 22 de enero de 2014 Revisado: 22 de marzo de 2014 Aceptado: 27 de junio de 2014

Nancy Carolina Melo Jaramillo**

Secretaria de Educación Distrital de Bogotá

\section{RESUMEN}

La pretendida educación de calidad, gratuidad y pertinencia, objetivos de la políica educativa implementada en Bogotá, no se ha logrado a pesar de constituirse en elemento fundamental de los planes de desarrollo implementados en el transcurso de los últimos 10 años. Su más grande impedimento es la escasa participación de docentes y directivos docentes de las instituciones de educación básica y media en razón a la vigencia de una estructura de mando piramidal en la que a estos actores solo le llegan órdenes y directrices que cumplir sin que su activa participación se haga efectiva. La investigación que da origen a este artículo se fundamenta, desde lo teórico en las tesis de sujetoactor social de Alain Touraine que permite construir

\footnotetext{
* Artículo de investigación.

** Correspondencia: Nancy Carolina Melo Jaramillo. Licenciada en Biología. Magister en Calidad y Gestión Integral. Docente de la Secretaría de Educación Distrital de Bogotá, Colombia. Correo electrónico: ncmj9@yahoo.es
} 
formas de participación en la gestión educativa; desde lo epistemológico en el paradigma positivista que permite su desarrollo a través de momentos sucesivos de planificación, ejecución, evaluación y comunicación de resultados; desde lo metodológico se aplica el método inductivo-deductivo, para conocer la realidad particular de la Institución Educativa Distrital General Santander de la ciudad de Bogotá, en este sentido el estudio adquiere las características de un estudio piloto. Los hallazgos de la investigación permiten formular lineamientos para el logro de los objetivos de la política educativa a través de una efectiva participación de los docentes.

Palabras clave: actores sociales, participación activa, gestión integrada, certificación, sistema de gestión de la calidad.

\section{ABSTRACT}

The alleged quality education, free and relevance, objectives of the educational policy implemented in the Capital District of Bogota, have not been achieved in spite of becoming a key element of development plans implemented during the past 10 years. Their biggest obstacle is the low participation of teachers and senior teachers of primary and secondary education institutios, due to the validity of a pyramidal structure in which these actors alone will come to meet orders and guidelines without active participation becomes effective. The research that gives rise to this article is based, from the theoretical in the thesis of subjectsocial actor of Alain Touraine for building forms of participation in educational management; from the positivist epistemological paradigm which allows its development through successive stages of planning, execution, evaluation and communication of results; from methodological, inductive-deductive method is applied to determine the particular reality of the district educational institution General Santander in Bogotá, in this sense, it acquires the characteristics of a pilot study. The findings of the research allows formulating guidelines for the achievement of the objectives of education policy through effective participation of teachers.

Keywords: Stakeholders, active participation, integrated management, certification, quality management system.

\section{INTRODUCCIÓN}

\section{El problema de la "No Participación"}

A pesar de los mandatos constitucionales, normas legales y actos administrativos que así lo establecen, no se han logrado cumplir los objetivos de la política de educación distrital por la inefectiva participación de docentes y directivos docentes de las instituciones de educación del Distrito, fundamentalmente en las instituciones de básica, secundaria y media. Parece evidenciarse una falsa apreciación de los directivos del nivel central, es decir los de la Secretaría de Educación Distrital (SED), que lograr la certificación del sistema de gestión de la calidad es un requisito formal que posiciona a esta institución y a las Instituciones Educativas del Distrito (IED) que dependen de ella, en un lugar privilegiado del mundo de la educación, sin tener en cuenta que esta falsa apreciación impide el desarrollo de principios y la promoción de programas y actividades en búsqueda permanente del mejoramiento de su calidad y excelencia, objetivo fundamental de la política trazada.

La estrategia que se sigue así lo evidencia, en efecto, la estructura de mando en el sistema educativo distrital privilegia un sentido piramidal, de arriba hacia 
abajo, de los cuerpos del nivel central de la SED a los docentes y directivos docentes de las instituciones educativas, a quienes solo les llegan instrucciones para cumplir e informes que presentar, sin ninguna participación activa ni efectiva en el proceso de toma de decisiones. Esta invisibilización genera apatía y desmotivación que impide la obtención de los objetivos de la política educativa trazada en los planes de desarrollo distrital.

En este artículo se presentan no solo un diagnóstico de la participación de estos importantes actores en las decisiones que tienen que ver con la revisión, evaluación, ajustes y reformulación de la política educativa distrital, teniendo en cuenta la percepción que ellos tienen de esa participación en el momento, sino también una serie de lineamientos que se requieren para la obtención de una activa y efectiva participación en los procesos que la calidad y la gestión integral de la educación exige para el logro de los objetivos de la políica formulada.

Solo de esta forma se logra modificar la tradicional estrategia de la pirámide del sistema educativo en la que las decisiones y acciones tienen un sentido que va del nivel central hacia la base, para orientarla en forma tal que permita concitar la activa y efectiva participación de directivos docentes y docentes de las instituciones en el proceso hacia la participativa integralidad de los sistemas de gestión. En este sentido es que a estos se les asume como sujetos y actores sociales válidos para ser consultados sobre su ejercicio profesional y sus acciones para alcanzar los objetivos de la políitca educativa.

Ahora, el desconocer las repercusiones que tiene la obtención de la certificación de calidad en la SED, sin la participación activa y efectiva de los docentes y directivos docentes de las instituciones de educación básica secundaria y media de Bogotá, hace que se deje de lado el desarrollo de los principios de gestión de la calidad para la rama ejecutiva del poder público y la promoción de programas y actividades en búsqueda permanente del mejoramiento de esa calidad ya certificada. Esto en razón a la falsa apreciación de que el cumplir formalmente con el requisito de certificar su gestión de calidad es el fin último, que abre puertas y posiciona a la Secretaría Distrital de Educación y a todas sus IED en un lugar privilegiado del mundo de la educación y que no hay necesidad de auscultar permanentemente la realidad educativa con el fin de obtener los objetivos de la política educativa, que apunten hacia la excelencia en el servicio. La estrategia se supedita entonces a trazar directrices y emitir órdenes para el mantenimiento de lo existente, sin la participación activa y efectiva de quienes en razón a su ejercicio profesional tienen permanente contacto con la realidad educativa local, conocen sus obstáculos e inconvenientes y están capacitados para formular e implementar posibles soluciones.

En la investigación en la que se sustenta este artículo, se adelanta un acercamiento a docentes y directivos docentes de una de estas instituciones -el Colegio General Santander de la localidad de Engativa-, a partir de la observación directa y la aplicación de una encuesta como instrumento que permite identificar percepciones respecto de su participación en la gestión educativa que adelanta la SED y la forma en que consideran se debe adelantar dicha gestión para poder lograr los objetivos de la política educativa. En este sentido, la investigación se constituye en un estudio piloto que marca la ruta para la indagación futura a una muestra representativa de todas las organizaciones de las distintas localidades de Bogotá.

Los lineamientos que se formulan más adelante y que tienen como fin la construcción de un Modelo Participativo de Gestión integral de la educación en el Distrito Capital permiten, a través de su futura 
implementación, articular debidamente las acciones entre los directivos del nivel central con los del nivel local a través de la efectiva participación de estos importantes actores.

La importancia de la investigación radica en la no existencia de investigaciones similares conocidas en el ámbito nacional ni latinoamericano. Solo existen aquellas realizadas por investigadores al interior de la Maestría en Calidad y Gestión Integral del Convenio USTA - ICONTEC en Colombia, en las que se resalta la participación del personal docente como uno de los componente más importantes para que un colegio pueda alcanzar los objetivos asignados para la educación, "ya que son los docentes quienes desarrollan la mayor parte de las acciones del proceso de enseñanza - aprendizaje y son quienes tienen la responsabilidad y visibilidad ante los estudiantes y padres de familia de cada una de las etapas y logros que los primeros alcancen” (Acero y Ramírez, 2009, p. 187). En ese aspecto se encuentra la investigación del mismo posgrado que manifiesta que "Es importante tener en cuenta que existe interrelación tanto entre los elementos que componen el sistema educativo, como entre éste y la sociedad de la que forman parte. Esta última relación hace que el sistema educativo sea caracterizado como social y abierto y esto en el marco del concepto de sistema, se convierte en el transformador de insumos provenientes de la sociedad" (Pedraza, 2010, p.63).

\section{METODOLOGÍA}

En el plano teórico, la propuesta de lineamientos para la construcción de un modelo participativo de gestión integral de la educación en la ciudad de Bogotá, acorde con el mandato de la política educativa trazada por la SED, se apoya en el Paradigma Sistémico de Edgar Morin y en los planteamientos de Alain Touraine sobre la configuración del "Sujeto" y del "Actor Social", para validar la participación activa y efectiva de docentes y directivos docentes de las instituciones en el proceso hacia su elaboración o construcción.

En efecto, de acuerdo con Morin citado por Cunha, "una institución bien gestionada no se preocupa por acumular el saber sino en disponer simultáneamente de una actitud general para plantear y analizar problemas y principios organizadores que permitan vincular de manera sistémica los saberes y darles sentido, desde una auto-eco-organización que no simplifique las inter-retroacciones sino que permita articularlas y asociarlas" (1989, p. 23), actitud que se obtiene a través de la eficiente participación de sus actores. El develar los intangibles que identifican las razones que impiden la existencia de una actitud general para proceder como actores sociales, es decir, para plantear y analizar problemas por parte de directivos docentes y docentes, en este caso de la IED General Santander, y argumentar sobre sus posibles soluciones, facilita formular lineamientos y principios organizadores vinculando de forma sistémica saberes que conduzcan a acciones y retroacciones articuladas y asociadas en búsqueda del propósito común de obtener el logro de los objetivos de la política educativa.

De acuerdo con Touraine (1997), las personas se configuran como "Sujetos" cuando tienen una participación activa en las decisiones que afectan su vida personal y sus proyectos de vida, y como "Actores sociales" cuando tienen una participación efectiva en las decisiones que afectan la vida de entorno colectivo en que ellos se encuentran inmersos. En este sentido se comportan como "Sujetos" cuando adquieren la capacidad de asumirse a sí mismos como actores de su propia vida, es decir, cuando son "capaces de tener proyectos, de elegir, de juzgar de modo positivo o negativo, y capaces también, más sencillamente, de tener relaciones sociales, ya se trate de relaciones de cooperación, de consenso 
o conflictivas" (Touraine, 1997, p. 41). El mencionado autor también explica que se asumen como "Actores sociales" cuando además de ser protagonistas de su propia transformación se configuran como protagonistas de la transformación del entorno en el que están inmersos, caso en el cual, además de tener objetivos personales, expresar lo que percibe en sí mismo y en los otros, o capacidad de comunicación con los demás, debe tener conciencia ciudadana o capacidad de diálogo y participación efectiva.

La configuración de la persona como "Actor social" está determinada entonces por la conciencia ciudadana que solo se adquiere cuando la persona es consciente de que su participación es efectiva, es decir, cuando además de la activa participación y expresión de sus opiniones, estas son tenidas en cuenta y forman parte de las decisiones de gobierno y de su implementación y evaluación, situación que no se presenta en las instituciones educativas del Distrito Capital.

En esta investigación se asume al directivo y al docente de las instituciones educativas de Bogotá como "Sujeto" y como "Actor social". Como "Sujeto" se asume integrados a su comunidad académica en la que ejercen sus funciones, pues está en capacidad de actuar, opinar, contradecir, argumentar y aspirar a contribuir al fortalecimiento de la cultura educativa y académica de su institución y hace posible la vinculación de su pasado y su futuro, su historia personal y laboral y su situación colectiva, tiene un proyecto de vida laboral y personal que aspira a realizar. Como "Actores sociales" se asumen protagonistas del desarrollo de la comunidad académica en la que laboran y de la sociedad en la que se encuentran en el día a día; o sea, como individuos que no solo tienen sino que intentan realizar objetivos y proyectos personales en un entorno constituido por otros actores, un "entorno que constituye una colectividad a la que él siente que pertenece y cuya cultura y reglas de funcionamiento institucional hace suyas, aunque solo sea en parte" (Touraine, 1997, p. 43).

Ahora, en relación con su participación, esta es una participación activa cuando en ejercicio de su capacidad para comunicarse, tomar decisiones y argumentar, los directivos docentes y docentes se comportan como "sujetos" y es una "participación efectiva" cuando esas capacidades de participación y aporte son reconocidas y se tienen en cuenta para tomar decisiones respecto de la gestión educativa en el ámbito de las instituciones educativas como en el ámbito del nivel central, es decir, por los directivos de la Secretaría de Educación (López, 2009). En una palabra, su participación activa y efectiva, en forma simultánea, se constituye en elemento cohesionador de la gestión integral adelantada para obtener los objetivos de la política educativa y los principios de la gestión de calidad contenidos en la norma técnica NTCGP1000 (Departamento Administrativo de la Función Pública- DAFP, 2009).

Contrario a la participación efectiva, es la participación formal, la que se asume en este estudio como aquella que se adelanta con el fin de cumplir un requisito 0 un mandato normativo sin que las propuestas, críticas 0 argumentos de los participantes, generalmente citados por un superior jerárquico, tengan algún efecto en las decisiones 0 acciones que se adelanten en el futuro. Según López (2009), también es contraria la No Participación, entendida como la ausencia total de participación derivada de la apatía o la no motivación de una persona en relación con la temática a tratar o la acción a emprender en un determinado evento al que ha sido invitado o citado previamente. La no participación también se deriva de la intencionada exclusión 0 no invitación a participar de parte de quienes organizan la agenda, las invitaciones o citaciones, sin que genere ninguna reacción, por parte del excluido, en contra de la decisión. 
Metodológicamente el estudio busca la formulación de lineamientos para la construcción de un Modelo Participativo para la Gestión Integral en la IED General Santander de Bogotá, cuya implementación garantice la perdurabilidad o permanencia del futuro Sistema de Gestión Integral a través de trascender la estrategia de la pirámide de arriba hacia abajo para pasar a ser de abajo hacia arriba. Para ello se apoya inicialmente en información secundaria, posteriormente en información primaria derivada de la elaboración de una matriz teórica en la que se cruzan los Principios de la norma NTCGP1000 con las características de la gestión de la educación adelantada en la Secretaría de Educación Distrital, articuladas por las formas de participación o no participación de docentes y directivos docentes de la IED General Santander. Esto permite construir indicadores de participación para poder construir una encuesta a través de la cual se identifica y se mide la participación que en la realidad actual (diagnóstico) evidencian directivos docentes y docentes del colegio, y el deber ser de la participación, de acuerdo a la percepción de los encuestados, en el proceso hacia la implementación, certificación e integralidad de los sistemas de gestión. La encuesta se somete a un estudio piloto que hace posible identificar y ajustar los interrogantes a aplicar, el cual se realiza en el momento anterior al trabajo de campo. Los lineamientos para la construcción de un Modelo Participativo para la Gestión Integral que se formulan, garantizan la perdurabilidad de los subsistemas que integrarían el futuro Sistema de Gestión Integral y el logro en últimas de los objetivos de la política educativa del Distrito.

\section{RESULTADOS Y DISCUSIÓN}

En relación con el diagnóstico de la participación en la política educativa se encuentra que existe una extraordinaria descoordinación entre la SED y las instituciones de educación, representadas en este caso piloto por la IED General Santander en el Distrito Capital de Bogotá, con respecto a la gestión que se desarrolla para la obtención de los objetivos de la política educativa distrital. Ello se evidencia en los siguientes factores:

\section{Conocimiento y participación en la política educativa distrital}

Es sobresaliente el desconocimiento que se evidencia en docentes y directivos docentes de la IED General Santander respecto de los objetivos de la política educativa formulada para el Distrito Capital de Bogotá $(38,53 \%)$. Este desconocimiento es mucho más sobresaliente si se tiene en cuenta que en ambas jornadas, mañana y tarde, más del $45 \%$ de los encuestados sobrepasa los diez años de vinculación a la institución, tiempo más que suficiente para conocer la evolución de la política educativa formulada por la SED. Se podría pensar que esta No Participación es más sobresaliente en aquellos directivos docentes y docentes que están pendientes de su pensión o de aquellos que habiéndola recibido continúan prestando sus servicios, lo que se podría reflejar en una desmotivación hacia el conocimiento de los objetivos de la política derivada de su próximo retiro, pero este supuesto no se evidencia en razón a que el porcentaje de quienes sobrepasan los 25 años de vinculación laboral al sistema educativo es mínimo $(7,46 \%)$.

El desconocimiento de la política educativa por parte de directivos docentes y docentes, actores fundamentales de la acción educativa, se deriva de inadecuados procesos de participación en estos aspectos. En efecto, se evidencia una No Participación cuando la mayor parte de los encuestados expresan no haber sido invitados a participar de la revisión de la política educativa $(73,39 \%)$ siendo más sobresaliente en los de la jornada de la mañana $(81,48 \%)$ o cuando consideran 
que no tienen tiempo $(0,92 \%)$ o tienen otros compromisos $(0,92 \%)$. Igualmente se identifican: una escasa Participación Activa pero no efectiva cuando a pesar de participar y argumentar propuestas estas no se tienen en cuenta $(14,68 \%)$ y una Participación Formal cuando asisten por cumplir la invitación que hacen directivos de la SED sin hacer mayores aportes $(10,09 \%)$.

Pero si para la revisión de la política educativa no se tiene en cuenta la participación de directivos docentes y docentes de la IED General Santander, tampoco para participar de la recolección y entrega de información valiosa, en su poder, para la revisión y formulación de la política educativa, participación que se hace obligante en su calidad de actores sociales clave del proceso educativo. En efecto, se evidencia una No Participación cercana al $91 \%$ de los docentes y directivos docentes en este proceso por el hecho de no ser invitados a participar $(73,39 \%)$ y por abstenerse voluntariamente de hacerlo (16,51\%), situación que se hace más representativa en la jornada de la tarde en donde la No Participación llega al $93 \%$ de los encuestados. Se evidencia también una Participación Formal cuando se asiste por cumplir (5,50\%) y un Participación Activa pero no efectiva cuando se asiste y se argumentan propuestas pero estas no son tenidas en cuenta $(3,67 \%)$.

\section{Conocimiento y participación}

\section{en la estructura del sistema educativo}

Es altamente significativo el desconocimiento que se evidencia en directivos docentes y docentes encuestados de la IED General Santander (42,20\%) sobre la estructura del sistema educativo distrital en razón a que ellos se constituyen en elementos fundamentales de la misma, sin cuya efectiva participación las actividades y procesos educativos no adquieren la calidad ni los desarrollos requeridos. Este desconocimiento se percibe mayoritariamente en los funcionarios de la jornada de la mañana (45\%) que en los de la tarde $(38,89 \%)$.
Pareciera obvio que un desconocimiento de tal naturaleza se derive en la No Participación de una proporción semejante de directivos docentes y docentes en la revisión de la estructura del sistema educativo, pero el alto porcentaje de quienes No Participan, superior al $98 \%$ en ambas jornadas, limita el anterior supuesto. Se puede suponer, igualmente, que el desconocimiento de la estructura del sistema educativo se presente en aquellos directivos docentes y docentes vinculados recientemente a sus labores educativas, pero el hecho de que más del $45 \%$ de los encuestados sobrepase los diez años de vinculación deja sin piso esta afirmación.

La razón de esta alarmante No Participación en la revisión de la estructura del sistema educativo distrital, obedece al hecho de que más del $96 \%$ de los directivos docentes y docentes de la IED General Santander objeto de este estudio piloto, no son invitados a participar, invisibilización que deriva en la percepción generalizada de que en el orden central, es decir en la SED, le dan poca importancia a su participación en los procesos hacia la toma de decisiones. En efecto, más del $60,55 \%$ de los encuestados perciben que los directivos del orden central toman las decisiones directamente sin reconocer importancia a los funcionarios de las instituciones a quienes consideran obligados a obedecer, mientras que cerca del $30 \%$ dice desconocer las razones de esta exclusión y el resto no muestran motivación alguna de querer participar.

\section{Conocimiento y participación en los sistemas de gestión}

Se presenta un alto desconocimiento de directivos docentes y docentes de la IED General Santander encuestados $(67,89 \%)$ respecto de los sistemas de gestión implementados por la SED, siendo los de la jornada de la mañana los que mayor desconocimiento tienen $(79,63 \%)$ en relación con los de la tarde $(56,36 \%)$. Esta situación es semejante a la presentada en relación 
con el desconocimiento de la política educativa y la estructura del sistema educativo distrital en el sentido de que el desconocimiento no procede de la existencia de un significativo porcentaje de encuestados recién vinculados al sistema educativo, en razón a que, como ya se hizo explícito, más del $45 \%$ lleva más de 10 años de vinculación, tiempo suficiente para tener un claro conocimiento sobre estos aspectos.

Los resultados del análisis de la información recolectada permiten colegir que la No Participación en la construcción o diseño, implementación y evaluación de sistemas de gestión vigentes en la SED obedece a la exclusión o invisibilización que desde el nivel central se tiene de directivos docentes y docentes de las instituciones educativas. En efecto, la casi totalidad de los encuestados $(94,50 \%)$ no es invitada por los directivos del nivel central a participar del diseño, implementación o evaluación de los sistemas de gestión existentes en la SED o no aceptan participar, situación que se hace crítica en los docentes y directivos docentes de la jornada de la mañana en donde el $100 \%$ manifiesta no ser invitado, en relación con los de la jornada de la tarde $(87,27 \%)$.

Respecto de la Participación Activa pero No Efectiva, es mínima dado solo el 3,67 \%) de los encuestados manifiesta que sí participa con argumentos y propuestas pero que estos no se tienen en cuenta $(3,67 \%)$, mientras la Participación Efectiva es imperceptible en razón a que solo el $0,92 \%$ expresa que participa activamente y que sus propuestas son tenidas en cuenta en el nivel central.

\section{Conocimiento y participación en los procesos adelantados}

El desconocimiento de los procesos que se adelantan en el sistema educativo distrital por parte de directivos docentes y docentes de la IED General Santander de
Bogotá es crítico en razón a la importancia que ellos deben tener como elementos fundamentales del sistema. En efecto, dado que el desconocimiento se manifiesta en más del $73 \%$ de los encuestados, proporción más o menos similar para las dos jornadas de trabajo, es poco lo que se puede esperar en la obtención de los resultados esperados en la política educativa contenida en los últimos planes de desarrollo.

Esta situación evidencia una generalizada No Participación en los procesos que se adelantan en el sistema educativo distrital fundamentalmente por el hecho de no ser invitados a participar (93\%), situación que se hace más crítica en los docentes y directivos docentes de la jornada de la mañana (98\%) en relación con los de la tarde (89\%). La casi imperceptible Participación Activa pero no efectiva se evidencia en quienes siendo invitados a participar en los procesos y presentando y argumentado propuestas para su calidad $(3 \%)$, no son tenidas en cuenta por quienes toman las decisiones en la SED.

Se evidencia una muy fuerte desmotivación de los docentes y directivos docentes a participar de los procesos del sistema educativo distrital, quienes aluden que no son invitados en razón a que las decisiones son tomadas directamente por el nivel central sin tenerlos en cuenta $(45,87 \%)$, otro porcentaje representativo manifiesta desconocer las razones para no ser invitados $(31,19 \%)$; el resto no se considera con la importancia suficiente como para ser tenido en cuenta por el nivel central, los directivos docentes del nivel central no los consideran importantes o no consideran necesaria su participación en una clara muestra de desmotivación.

Respecto de la relación que deben tener la estructura del sistema educativo distrital, los sistemas de gestión implementados y los procesos que se adelantan en el sistema educativo, obviamente, si se evidencia 
un amplio desconocimiento de docentes y directivos docentes sobre la política formulada por la SED, se colige que es amplio también el desconocimiento sobre la relación que tienen o deben tener cada uno de estos aspectos para el cabal funcionamiento del sistema, situación que se evidencia en más del $81 \%$ de los encuestados, y se hace más compleja en directivos docentes y docentes de la jornada de la tarde $(87,27 \%)$ en relación con los de la mañana $(75,93 \%)$.

\section{Cumplimiento de principios para una educación de calidad}

A manera de síntesis de todas estas conclusiones respecto de la percepción de los docentes y directivos docentes en relación con su conocimiento sobre la política educativa distrital y su participación en la entrega de información para su revisión y ajuste, se puede afirmar que no existe una activa y efectiva participación que garantice la toma de decisiones relacionadas con el "enfoque al cliente" es decir, a los jóvenes a quienes se orienta en servicio educativo por parte del Distrito, actores fundamentales de la política educativa. En efecto, son los docentes y directivos docentes quienes se constituyen en las personas más autorizadas, en relación con su cercanía a los jóvenes de las comunidades en donde prestan sus servicios, para establecer si los objetivos de la política en relación con la calidad del servicio están relacionados con las necesidades, son ellos también quienes deben transmitir información sobre las necesidades y expectativas del servicio en búsqueda de un mejoramiento continuo.

En este sentido, las decisiones de la SED parecen no orientarse mucho por el análisis de datos y de la información suministrada de primera mano por quienes, en el ejercicio de sus funciones educativas, están en contacto directo con los usuarios del servicio y portadores del derecho a la educación, lo que limita el fortalecimiento de su capacidad para demostrar la eficacia de las decisiones anteriores mediante la referencia a los registros de los hechos.

Ahora, el muy escaso conocimiento de docentes y directivos docentes sobre los sistemas de gestión implementados en la SED y su no efectiva participación en los procesos de revisión y evaluación hacen muy difícil identificar, entender y gestionar procesos interrelacionados como un sistema y afecta la eficacia y eficiencia del sistema educativo distrital en el logro de los objetivos formulados en la política educativa. Esto, ante la dificultad que se presenta para integrar y alinear estos procesos de tal forma que permitan obtener los resultados deseados en la política, y ante la desconfianza que se genera en las partes interesadas con relación a la coherencia, la eficacia y la eficiencia de ellos.

En síntesis la no participación efectiva de directivos docentes y docentes en los distintos procesos que se adelantan en el sistema educativo distrital en relación con la política educativa impide la obtención de los objetivos, hace imposible la predicción de sus resultados y minimiza las oportunidades de mejora centradas y priorizadas.

En relación con el liderazgo que debe asumir la SED en la gestión del sistema educativo distrital, se evidencia debilidad en el cumplimiento de su responsabilidad de asegurar la unidad de propósito y orientación; en este sentido, no logra el involucramiento general de docentes y directivos docentes de la IED General Santander en la obtención de los objetivos de la política educativa formulada en sus planes de desarrollo. En efecto, la SED no transmite objetivos y metas claras, ni logra mantener valores compartidos ni establecer confianza y eliminar temores, tampoco logra inspirar, fomentar y reconocer la contribución de docentes y directivos docentes a la obtención de estos objetivos. 
Por último, para la SED no parece ser un objetivo permanente la mejora continua en el sistema de educación distrital y en su organismo rector. En efecto, el desconocimiento y la muy escasa participación de docentes y directivos docentes del Colegio General Santander, de los procesos más importantes que se adelantan en relación con el servicio educativo, impide el mejoramiento de las capacidades del sistema educativo distrital, lo que genera desventajas en su desempeño, dificulta la alineación de las actividades y procesos de mejora con los objetivos estratégicos formulados en la política y obstaculiza la flexibilidad necesaria para reaccionar rápidamente a las oportunidades de brindar un servicio de calidad y excelencia.

\section{CONCLUSIONES}

Del estudio realizado, es posible definir algunos lineamientos para la construcción de un modelo participativo de gestión integral.

\section{Del enfoque al cliente}

Aplicar el principio del enfoque al cliente debe conducir hacia la comprensión de las necesidades y expectativas del estudiante de la IED General Santander y en general de las instituciones de todas las localidades distritales. Esto con el fin de asegurar que los objetivos de la política educativa estén relacionados con las necesidades y expectativas de los estudiantes, las que se hace imposible conocer sin la participación de sus docentes y directivos docentes, dado que son ellos los que poseen de primera mano la información. Esto exige la formulación de estrategias que les permita a estos actores no solo medir la satisfacción de estas necesidades y proceder acorde con los resultados obtenidos sino comunicarlas con el fin de que la SED proceda a gestionar de manera adecuada las relaciones instituciones - estudiantes. En últimas, ello asegura la existencia de un enfoque equilibrado entre la satisfacción de necesidades educativa y las partes interesadas beneficiando a la comunidad inicialmente y a toda la sociedad en general.

\section{Del liderazgo de la SED}

La Secretaría de Educación Distrital debe ejercer el liderazgo que viene perdiendo en razón a que no logra establecer la unidad de propósito y orientación en el sistema educativo distrital que les permita a todas las partes interesadas, principalmente a docentes y directivos docentes de las distintas instituciones educativas, la motivación necesaria para su involucramiento en el logro de los objetivos de la política educativa. Para ello debe considerar no solo las necesidades de los estudiantes sino las expectativas de directivos y docentes como miembros importantes de las partes interesadas. Además, debe establecer confianza y mitigar temores, impulsar la creación de valores compartidos, proporcionar los recursos necesarios para lograr que estos actúen participativa y responsablemente reconociendo y fomentando sus contribuciones.

\section{De la participación en los procesos}

La generalizada No Participación en los procesos que se adelantan al interior de la IED General Santander de Bogotá exige definir de manera sistemática, por parte de la SED, las actividades a seguir para obtener los resultados esperados de la implementación de la política educativa. Para ello es conveniente invitar a participar, pues es importante para todo el sistema educativo distrital. Solo así se puede asignar responsabilidades para su cabal desarrollo teniendo en cuenta las capacidades, formación y experiencia, definir participativamente los recursos requeridos para su cabal cumplimiento y evaluar riesgos, efectos e impactos en todo el proceso. 


\section{De la participación en los sistemas de gestión}

Dado que los sistemas de gestión permiten la interrelación de los distintos procesos que se adelantan en el sistema educativo distrital, la eficacia y eficiencia de todo este sistema en el logro de los objetivos de la política educativa se afecta extraordinariamente por la № Participación generalizada y la escasa participación no activa ni efectiva de docentes y directivos docentes de las instituciones educativas. Es indispensable entonces comprender por parte de la SED las interdependencias de todos y cada uno de los procesos que se adelantan en una permanente alimentación y retroalimentación de arriba hacia abajo y de abajo hacia arriba de la pirámide del sistema educativo distrital. No se trata de proseguir con la orientación actual de la estructura del sistema, en el que los docentes y directivos docentes reciben órdenes de la SED que tienen o deben cumplir sin participar efectivamente del proceso de toma de esas decisiones, tampoco se trata de invertir el sentido como se planteaba originalmente en el proyecto de investigación, sino de compartir, de retroalimentarse mutuamente y de tomar decisiones derivadas de un proceso efectico de participación de todas las partes interesadas, fundamentalmente de directivos de la SED y docentes y directivos decentes de las instituciones educativas. Para ello también es indispensable la participación efectiva de todos estos actores en la medición y evaluación de la polííca educativa y en la determinación de los ajustes a realizar.

\section{De la mejora continua}

Se plantea la necesidad de que la mejora continua, objetivo fundamental de la política educativa, no se deje de lado o no se confunda en la SED con el proceso de certificación de calidad obtenido en esta dependencia. En este sentido obtener la certificación de calidad no se debe asumir como el objetivo fundamental de la polííca, al contrario, es un elemento más que permite, aunado a otros esfuerzos, un permanente tránsito hacia la mejora continua del sistema educativo distrital.

La mejora continua debe ser un concepto integral cuya aplicación debe mejorar el desempeño de todo el sistema educativo, proporcionar a docentes y directivos docentes los métodos y las herramientas participativas para superar en calidad el logro de los objetivos de la política educativa respecto del servicio educativo que brinda, y establecer metas que guíen el proceder de las partes interesadas hacia esa mejora. Un aspecto importante a seguir por parte de la SED es admitir y reconocer la mejora continua y los aportes de docentes y directivos docentes para su logro permanente.

\section{De los hechos para la toma de decisiones}

Evidentemente las posibles decisiones eficaces para el sistema educativo distrital, o mejor, las decisiones de política educativa en Bogotá, se deben fundamentar en el análisis de los datos y de la información, los que para poder adquirir la confiabilidad y exactitud requerida deben proveer de quienes los obtienen de primera mano, es decir, de los docentes y directivos docentes de las instituciones educativas de Bogotá. Hacer los datos accesibles para los directivos de la SED implica la participación efectiva de estos actores no solo en el proceso de suministro sino en el de análisis compartido y en el de toma de acciones basadas en esos análisis. Su afectiva participación, se reitera, es derivada de su experiencia y contacto permanente con los "clientes" y demás grupos de interés del sistema educativo.

\section{De las relaciones mutuamente beneficiosas con el proveedor}

Ya se han establecido lineamientos relacionados con la satisfacción de las necesidades y requerimientos de los estudiantes en el sistema educativo distrital, en el que la participación efectiva de los docentes y directivos es 
indispensable en su calidad de tener contacto directo con los estudiantes y demás actores del sistema. Pues bien, ese conocimiento es el que exige su participación, también efectiva, en la selección de materiales de apoyo pedagógico y temático que suministran los proveedores. Son ellos los que validan sus beneficios en el aporte hacia la obtención de los objetivos de la política educativa distrital. Su participación debe permitir contribuir en la identificación y selección de proveedores clave, compartir información, objetivos y metas de la políitica con miras a identificar los productos y servicios requeridos e inspirar, alentar y reconocer los logros obtenidos de los buenos proveedores.

\section{REFERENCIAS}

Acero, G. y Ramírez, J. (2007). Efectos de la certificación de calidad en la educación básica en el Distrito Capital (p. 187). Trabajo de Grado presentado como requisito parcial para obtener el título de Magister en Calidad y Gestión Integral del Convenio USTA - ICONTEC.
Cunha, F. (1989). Nocões de teoría de sistema (mimeo). Recife: Universidade Federal de Pernambuco.

Departamento Administrativo de la Función Pública, DAFP. (2009). Norma Técnica de Calidad en la Gestión Pública (p. 94). Bogotá: ICONTEC.

López, A. (2009). Construcción social de "juventud rural" y políticas de juventud rural en la Zona andina colombiana (p. 327). Tesis Doctoral. Buenos Aires, Argentina: Biblioteca virtual del Consejo Latinoamericano de Ciencias Sociales (CLACSO).

Pedraza, X. (2010). Aporte del enfoque sistémico del modelo de gestión de calidad ISO 9001 al modelo de acreditación institucional en instituciones privadas de educación superior. Trabajo de Grado presentado como requisito parcial para obtener el título de Magister en Calidad y Gestión Integral del Convenio USTA - ICONTEC.

Touraine, A. (1997). Juventud y democracia en Chile (pp. 36-48). En: Revista Última década. № 8. Valparaiso. 